\title{
Permanencia y cambio en la percepción de los peligros urbanos
}

\section{Permanence and change in the perception of urban hazards}

\author{
Judith Ley García' [D y Fabiola Maribel Denegri de Dios²
}

\begin{abstract}
RESUMEN
En este documento se exploran cambios y permanencias en la percepción de los peligros del entorno de los habitantes de la ciudad de Mexicali, México, de 2011 a 2016. El análisis toma como base un estudio previo que identificó una percepción amplificada de peligros como efecto de la manifestación física de éstos en el lugar, y auguraba la atenuación de la percepción conforme la oferta de estímulos disminuyera o cesara. Este trabajo parte de tal supuesto para contribuir al análisis de un trayecto más amplio sobre la percepción de riesgo en la ciudad con énfasis en su dinámica y temporalidad. Para ese propósito, se comparó la información de dos encuestas locales acerca de las amenazas que los habitantes reconocen en el lugar $y$, a partir de indicadores de cambio, se identificaron los peligros permanentes y cambiantes en la percepción social, así como su manifestación física en la ciudad. Producto de este análisis, se detectó una disminución importante en la percepción social que puede resultar en la reducción de medidas preventivas y prácticas de seguridad en los hogares urbanos.
\end{abstract}

Palabras clave: percepción de riesgo, peligros constantes, peligros cambiantes, riesgo urbano, Mexicali.

\begin{abstract}
This paper explores changes and permanence in the perception of environmental hazards of the inhabitants of the city of Mexicali, Mexico, from 2011 to 2016. The analysis is based on a previous study that identified an amplified perception of hazards as an effect of their physical manifestation at the site and predicted the attenuation of perception as the supply of stimuli decreased or ceased. This work takes into account this assumption to contribute to the analysis of a broader path on risk perception in the city with emphasis on its dynamics and temporality. For that purpose, the information from two local surveys about the dangers that inhabitants recognized in the place was compared and based on indicators of change, the permanent and changing hazards in social perception were identified, as well as their physical manifestation in the city. As a result of this analysis, a significant decrease in social which may result in the reduction of preventive measures and safety practices in urban homes.
\end{abstract}

Keywords: risk perception, constant hazards, changing hazards, urban risk, Mexicali. 
El incremento en el número de desastres relacionados con peligros naturales y antropogénicos despertaron el interés mundial en los países en desarrollo, sobre todo, a partir de que se proclamó la década de los años noventa como el Decenio Internacional para la Reducción de Desastres Naturales (ONU, 1987).

Desde el Decenio, diversos esfuerzos internacionales se han dirigido a reducir los impactos negativos de los fenómenos naturales (ONU, 1994; 2005; 2015a); sin embargo, el recuento de eventos, daños y pérdidas ha hecho evidente que "el riesgo de desastres a nivel mundial sigue acumulándose más rápido de lo que se reduce" (ONU, 2015b: 3/23), y se presenta principalmente en los países en desarrollo, cuyas ciudades enfrentan amenazas sin precedentes y se han convertido en sitios de magnificación de desastres (Estrada, 2009; ONU, 2017).

Las ciudades, además de aglutinar gran parte de la población mundial, actividades e inversiones, se encuentran expuestas a múltiples peligros, cuya acción individual o combinada encuentra un amplio abanico de posibilidades de daño y afectación. Cada porción del territorio posee un conjunto particular de amenazas (Cutter, 1993) y alberga una población que, por sus características, tiene distintas vulnerabilidades o capacidades para "anticipar, enfrentar, resistir y recuperarse del impacto" de ellas (Wisner et al., 2004: 11). De ahí que vulnerabilidad denota "debilidad, carencia o limitación de la comunidad, por tanto es definida como una incapacidad" (Cardoso, 2020: 140) que se produce socialmente y no puede desvincularse del peligro al que se está expuesto.

De tal manera que, si existe la posibilidad de que la acción individual o combinada de los peligros alcance a una población vulnerable, se considera que ésta se encuentra en riesgo. Cuando tal situación puede provocar la seria disrupción del funcionamiento de la comunidad y el impacto esperado incluye la lesión o pérdida de vida de gran cantidad de personas, así como mermas materiales que exceden los recursos de la comunidad para enfrentar la situación, entonces se considera que la comunidad está en riesgo de desastre (EIRD, 2009).

En este sentido, la reducción del riesgo de desastre requiere de un conjunto de estrategias que incluye, además del inventario de peligros o amenazas presentes en el territorio, la valoración de las condiciones de fragilidad en que se encuentra la población, tomando en cuenta que:

“Los desastres pueden reducirse considerablemente si la población está bien informada y motivada para asumir una cultura de prevención y de resiliencia ante los desastres, lo que a su vez impone la necesidad de reunir, compilar y divulgar los conocimientos e información pertinentes sobre las amenazas, los factores de vulnerabilidad y la capacidad" (ONU, 2005:6).

En el extracto anterior Naciones Unidas asume que la población es un agente activo y puede ser la principal promotora de su seguridad, ya que la capacidad de prevención y respuesta se fortalece a medida que las personas saben qué hacer y están dispuestas a protegerse. Para ello, es indispensable que puedan identificar o tener conocimiento útil sobre el conjunto de peligros presentes en el lugar que habitan.

Así, la reducción del riesgo de desastre precisa de la percepción de riesgo de los individuos (Qasim et al., 2015) que, por su naturaleza subjetiva, frecuentemente se encuentra sesgada con 
respecto a la situación objetiva. Estos desfases perceptuales han sido denominados amplificación y atenuación, para referirse a las percepciones exagerada y reducida de peligros o riesgos, respectivamente (Kasperson \& Kasperson, 1996).

Los sesgos perceptuales influyen de manera directa en las estrategias y prácticas de seguridad de las personas y, por lo tanto, contribuyen en la vulnerabilidad, especialmente la atenuación, pues a medida que se disminuye el riesgo percibido, se reduce la necesidad de adoptar medidas preventivas y se amplían las posibilidades de padecer los efectos negativos directos e indirectos de los peligros existentes (Fielding et al., 2014).

Cabe destacar que la percepción no es homogénea entre los habitantes de un lugar ni se mantiene constante en el tiempo, sino que cambia espacial y temporalmente en relación con distintos aspectos (Ibuka et al., 2010; Ley et al., 2015; Ley et al., 2019), de tal forma que algunos peligros pueden mantenerse en la percepción de los habitantes y otros cambiar, transitando de estados de atenuación a amplificación o viceversa (Fielding et al., 2014; Ley et al., 2016).

A pesar de la importancia que tiene la temporalidad de la percepción de las amenazas del entorno, al contribuir en relajar o hacer más estrictas las prácticas de seguridad de las personas en un lugar concreto. Este aspecto de la percepción ha sido escasamente abordado en la literatura (Sadik et al., 2017) y su estudio se ha centrado principalmente en la observación de un peligro a la vez (Ibuka et al., 2010; Fielding et al., 2014; Mañez et al., 2016; Suzuki et al., 2018) sin considerar el paisaje urbano de amenazas.

En este sentido, con el objetivo de contribuir al conocimiento de la dinámica temporal de la percepción, el presente documento explora los cambios en la visibilidad urbana de múltiples peligros a partir de la información proveniente de dos encuestas locales de percepción (UABC, 2011, 2016) aplicadas en la ciudad de Mexicali en México.

La selección de la ciudad de Mexicali como área de estudio obedece a dos razones: la primera, porque su localización le expone a un paisaje complejo de amenazas cuya acción aislada o conjunta puede derivar en un estado de desastre, especialmente si los habitantes no las identifican o reconocen en el lugar. La segunda, porque un estudio previo (Ley et al., 2016) reporta los cambios perceptuales ocurridos en el quinquenio anterior (2006 a 2011), sirviendo como base de análisis del período subsecuente (2011 a 2016) que se reporta en este documento.

En tal estudio, las autoras señalan que, al final del periodo, se presentó un aumento considerable en la visibilidad social de los peligros y la amplificación de varios de ellos, como estado de alerta que despertó la manifestación física de varias amenazas en el lugar. Las autoras consideraron que, conforme la oferta de estímulos cesara, la percepción disminuiría paulatinamente. En este documento se aborda esta suposición como contribución al análisis de un trayecto más amplio sobre la percepción del riesgo en el lugar con énfasis en su dinámica y temporalidad. 


\section{La temporalidad en la percepción del paisaje de amenazas}

A partir de los años setenta se ha desarrollado una extensa literatura sobre percepción de riesgo, especialmente desde la psicología cognitiva, cuya atención se ha centrado en explicar las diferencias perceptuales entre expertos y públicos legos, así como entre distintos grupos de personas y peligros (Margolis, 1996; Slovic, 2000), pero con escasos trabajos que abordan su dinámica y temporalidad. Desde esta perspectiva, la palabra percepción alude a los procesos mentales mediante los cuales los sujetos adquieren, procesan y evalúan la información que captan del ambiente físico y comunicativo (Renn, 2005).

El término riesgo se refiere a la incertidumbre sobre efectos adversos derivados del encuentro de peligros con personas o comunidades vulnerables (Ley, 2011). Por ello, la percepción de riesgo es concebida como "el proceso a través del cual los sujetos reconocen los peligros, identifican sus capacidades para enfrentarlos (resistir y recuperarse) y evalúan su situación de seguridad en función de ambos aspectos (estiman la probabilidad de efectos negativos, daños y pérdidas)" (Ley et al., 2019: 74). Consiste en una evaluación subjetiva que generalmente distorsiona la situación existente y se desfasa con respecto a la evaluación objetiva de la seguridad que realizan los expertos.

Estos desfases, sesgos o distorsiones perceptuales se presentan en dos sentidos opuestos: la amplificación y la atenuación de la idea del riesgo, como percepción de un mayor y menor nivel de riesgo que el existente o reportado por expertos, respectivamente (Kasperson et al., 1988; Kasperson \& Kasperson, 1996).

En relación con el paisaje de amenazas del hábitat, la percepción aumentada del riesgo (amplificación) implica que los peligros son sumamente visibles para los habitantes y sus efectos son sobre estimados o exagerados; mientras que, en la percepción disminuida (atenuación), los peligros del entorno son poco visibles y sus efectos son minimizados o ignorados.

La percepción es dinámica, se alimenta cotidianamente de información o estímulos ambientales y comunicacionales (Kasperson et al., 1988; Pidgeon et al., 2003), de tal forma que peligros altamente reconocidos por la población pueden perder importancia con el paso del tiempo; mientras que otros, inicialmente desconocidos o ignorados, pueden ganarla (Ibuka et al., 2010; Fielding et al., 2014; Ley et al., 2016; Suzuki et al., 2018; Obenauer et al., 2018). En estas transiciones participa la forma en que la información sobre los peligros es captada y procesada por los sujetos perceptores, que es explicada desde la psicología cognitiva a partir de una serie de heurísticas que se desarrollan en contextos sociales y culturales particulares.

\section{Disponibilidad y temporalidad}

En el proceso de identificación y evaluación de las amenazas del entorno participan diversos mecanismos cognitivos o heurísticas que permiten simplificar la realidad para hacerla más accesible al entendimiento humano. Una de las más importantes es la disponibilidad o accesibilidad de información (Tversky \& Kahneman, 1973; Folkes, 1988), que se refiere a la existencia y facilidad con que los trazos de memoria pueden ser recuperados o traídos a la mente (MacLeod \& Campbell, 1992). 
Este mecanismo posibilita que los eventos accesibles en la memoria sean más fácilmente representados o construidos en la mente que los inaccesibles (Sherman et al., 1985), aumentando la probabilidad de que sean percibidos o descubiertos en el entorno. Esto puede conducir a que los peligros de manifestación frecuente en el hábitat sean más fácilmente reconocidos e imaginados que los de baja o nula frecuencia, e incluso hacerlos susceptibles de amplificación. No obstante, tal situación puede tener el efecto contrario cuando la constante exposición a estímulos e información provoca la saturación e indiferencia de los habitantes (Tversky \& Kahneman, 1973).

La disponibilidad o accesibilidad de información sobre amenazas se ve afectada por la forma en que se ancla (Tversky \& Kahneman, 1973; 1974), marca (Finucane et al., 2000) y rescata información de la memoria; es decir, por la representatividad heurística (Covello, 1983), que alude a las características de los eventos y el tipo de emociones relacionadas con ellos que contribuyen a su memorización y posterior recuperación.

Consecuentemente, entre más vívidos o sorprendentes sean los eventos más fácilmente serán recordados por las personas, como también las situaciones únicas que llaman la atención (Folkes, 1988; MacLeod \& Campbell, 1992; Romanus, 1996). En ello es importante tener en cuenta el papel que juegan los medios de comunicación cuando desvían la atención pública hacia eventos novedosos o catastróficos (Tyler \& Cook, 1984).

En otras palabras, la disponibilidad por recuerdo explota la experiencia y el sufrimiento directos de las personas con respecto a eventos acontecidos en la comunidad o en la red social, e inhibe la emergencia de otras escenas o imágenes, incluso las promovidas por los medios de comunicación (Gregory \& Satterfield, 2002; Pachur et al., 2012). De ahí que los peligros con potencial catastrófico (baja frecuencia y alta intensidad) o aquellos que han dejado una marca en la comunidad, se encuentren más accesibles en la memoria y sean recordados por más tiempo que los de tipo crónico (alta frecuencia y baja intensidad) (Slovic, 2000; Stoffle et al., 1991).

Debido a que la experiencia previa juega un papel importante en la facilidad con que se construyen o imaginan escenarios futuros de riesgo y en cómo serán juzgados por las personas (Tversky \& Kahneman, 1974; Stoffle et al., 1991), se debe considerar que ésta tiene como referente básico el espacio físico o territorio. Es decir, cuando las amenazas evaluadas forman parte del hábitat, el entorno se vuelve una unidad perceptiva que ofrece estímulos e informaciones y enmarca la experiencia cognitiva (Durand, 2008). Así, la experiencia directa, la memoria y la percepción de amenazas pueden ser influidas por la presencia, proximidad y manifestación física de los peligros en el lugar (Qasim et al., 2015).

En resumen, para entender la dinámica temporal de la percepción de riesgo de los habitantes urbanos es importante considerar que la memoria tiende a decaer en el tiempo (MacLeod \& Campbell, 1992). Sobre todo, cuando disminuye la oferta de estímulos o información sobre las amenazas del entorno, o bien, cuando acontecimientos de otra índole atraen fuertemente la atención.

No obstante, es posible que algunos peligros permanezcan disponibles, particularmente aquellos que han sido anclados mediante la experiencia directa y los que se manifiestan de manera particular en el lugar. Por ello, es conveniente establecer una conexión del fenómeno 
perceptual con lo que sucede en el espacio habitado, y no sólo con los procesos mentales o juicios sobre amenazas ajenas al lugar, como reportan la mayoría de los textos sobre percepción de riesgo.

\section{Materiales y métodos}

En su noción más básica "percibirse en riesgo involucra identificar el peligro y saberse vulnerable ante él" (Ley et al., 2016: 277-278). Por ello, la percepción de riesgo puede considerarse una capacidad de los sujetos para identificar las amenazas presentes en el lugar que pueden afectarlos severamente. Este trabajo se centra en la primera parte del proceso perceptual e implica que los habitantes urbanos reconozcan los peligros del entorno y que, con el paso del tiempo, éstos permanezcan constantes en la percepción.

Para explorar lo anterior, se tomaron como fuente de información las encuestas locales de percepción aplicadas en 2011 y 2016 a muestras aleatorias e independientes de 385 y 390 hogares de la ciudad de Mexicali, respectivamente (UABC, 2011, 2016). Las muestras se calcularon con la fórmula de Sheaffer, Mendenhall \& Ott (1987), con un error de estimación de 0.05 y un nivel de confianza de 0.95, para el total de viviendas particulares habitadas $(209,092)$ que reportó el censo del Instituto Nacional de Estadística y Geografía (INEGI, 2010) en las localidades que integran la mancha urbana.

Entre otras variables, las encuestas registraron los peligros que los habitantes identifican en la colonia o fraccionamiento donde viven. De ellas se obtuvo la lista de 31 peligros (Cuadro $N^{\circ} 1$ ), tomando en cuenta las amenazas con potencial catastrófico que el Centro Nacional de Prevención de Desastres (CENAPRED, 2018) y la Ley General de Protección Civil (2012) identifican en el territorio nacional.

Cuadro $N^{\circ} 1$.

Peligros incluidos en ambas encuestas

\begin{tabular}{|l|l|}
\hline Tipo de peligro & Peligro \\
\hline Geológicos & $\begin{array}{l}\text { sismo, flujo de lodo*, deslizamiento, agrietamiento del suelo, } \\
\text { hundimiento, licuefacción, maremoto*, erupción volcánica* }\end{array}$ \\
\hline Hidrometeorológicos & $\begin{array}{l}\text { Ciclón, } \\
\text { ción, tormenta eléctrica*, lluvia intensa o tromba, inunda- } \\
\text { calor extremo. }\end{array}$ \\
\hline Químico o químico-tecnológico & $\begin{array}{l}\text { Incendio, explosión, fuga o derrame de sustancia química y } \\
\text { radiación nuclear* }\end{array}$ \\
\hline Sanitario o sanitario-ecológico & $\begin{array}{l}\text { plaga*, epidemia, contaminación del aire, contaminación del } \\
\text { agua y contaminación del suelo }\end{array}$ \\
\hline Socio-organizativo & $\begin{array}{l}\text { Accidente aéreo*, accidente ferroviario, terrorismo* y disturbio } \\
\text { social }\end{array}$ \\
\hline
\end{tabular}

* Peligros con probabilidad nula o muy baja en la ciudad.

Fuente: Elaboración propia. 
Procedimiento:

1) A partir de los porcentajes de las personas que identificaron cada peligro se calcularon para cada año los siguientes indicadores sugeridos por Ley et al. (2016):

a) El coeficiente de correlación de Pearson (R) para corroborar la relación lineal de las percepciones de ambos años, como indicador de permanencia o cambio de la percepción en general. El coeficiente de determinación $\left(\mathrm{R}^{2}\right)$ para observar la influencia de 2011 en 2016;

b) El cociente de las percepciones en ambos años ( $C p=$ percepción 2016/percepción 2011) como indicador de la velocidad o ritmo de cambio en el periodo; $y$

c) El valor absoluto del cambio de posición de los peligros ( $\mathrm{P}$ = posición en 2016 - posición en 2011) como indicador de la pérdida o ganancia de importancia en el periodo.

2) Con base en los indicadores anteriores se clasificaron los peligros en dos grupos, los cambiantes y los permanentes en la percepción social:

a) Los peligros cambiantes o dinámicos son aquellos que se movieron notablemente en la percepción social durante el periodo; se desplazaron hacia arriba (ascendentes) o hacia abajo (descendentes) 4 o más lugares $(P \geq 4)$ y presentaron un ritmo acelerado de cambio ( $\mathrm{Cp} \leq 0.20$ en descendentes).

b) Los peligros permanentes o constantes son aquellos que presentaron una escasa o nula movilidad en la percepción $(C p \geq 0.8, P \leq 3)$ ya sea porque permanecieron visibles (visibilidad alta) o invisibles (visibilidad baja) en el periodo analizado.

3) De los anteriores perfiles de peligro se seleccionaron los extremos (los más y menos dinámicos) para documentar su manifestación física en la ciudad en el periodo de 2007 a 2016, así como su cociente de cambio (Co), con la intención de abarcar cinco años previos a cada encuesta.

a) Para la encuesta de percepción de 2011 se registraron los eventos de 2007 a 2011.

b) Para la encuesta de 2016, los eventos de 2012 a 2016.

\section{El caso de estudio}

Mexicali se localiza en el estado de Baja California al noroeste de México, colinda con el estado de California, Estados Unidos y es zona de cruce binacional de personas, vehículos y mercancías. La ciudad posee un paisaje de amenazas complejo (Cuadro $\mathrm{N}^{\circ} 1$ ) producto, tanto de su localización geográfica, como de su dinámica económica, social y urbana.

Por su ubicación fronteriza ha experimentado un crecimiento industrial importante, con la concentración, manejo y transporte urbano de cada vez más sustancias químicas peligrosas y, por ende, con el incremento del riesgo químico (UABC, AM \& SEDESOL, 2011). Asociados también a la urbanización se encuentran los peligros sanitarios, presentes en Mexicali de manera permanente, destacando a nivel nacional en contaminación del aire (INECC, 2017) y con la presencia de contaminación del suelo y del agua por basura, químicos y agroquímicos depositados en terrenos rústicos, drenes y canales atrapados por la expansión urbana (UABC, AM \& SEDESOL, 2011). 
Mexicali se localiza en una de las zonas de más alta sismicidad en México (CFE, 2008), y la condición del sitio facilita que un terremoto desencadene otras amenazas geotécnicas, aumentando el riesgo geológico (UABC, AM \& SEDESOL, 2011). Además, se asienta en el desierto de Sonora, que se caracteriza por: temperaturas extremas en invierno y verano; vientos fuertes; tormentas de arena; y aridez. A pesar de la escasa precipitación pluvial, la ciudad padece de inundaciones frecuentes producto de la infraestructura urbana inadecuada (UABC, AM \& SEDESOL, 2011).

\section{Resultados y discusión}

El resultado del primer indicador muestra una correlación fuerte en los dos años analizados ( $R=0.9285)$ y la influencia de 2011 en 2016 en un 86\% $\left(R^{2}=0.8621\right)$ como indicador de la permanencia del conjunto de peligros en la percepción social. No obstante, en el período disminuyó el número de peligros que los habitantes distinguen en el lugar (cuadro 2). Esto se observa en la reducción del porcentaje (de $29 \%$ a $5 \%$ ) de quienes identifican once o más peligros y en el aumento del porcentaje (de $30 \%$ a $62 \%$ ) de quienes observan pocos peligros (5 o menos) en el lugar.

Cuadro $\mathrm{N}^{\circ} 2$.

Porcentaje de personas por número de peligros que identifican en el lugar

\begin{tabular}{|l|l|l|}
\hline Peligros & 2011 & 2016 \\
\hline 0 & $0 \%$ & $7 \%$ \\
\hline 1 a 5 & $30 \%$ & $55 \%$ \\
\hline 6 a 10 & $41 \%$ & $33 \%$ \\
\hline 11 a 15 & $22 \%$ & $4 \%$ \\
\hline 16 a 20 & $6 \%$ & $1 \%$ \\
\hline 21 a 35 & $1 \%$ & $0 \%$ \\
\hline
\end{tabular}

Fuente: Elaboración propia.

En otras palabras, la percepción general de los peligros disminuyó de manera importante en el transcurso de cinco años, como sugirieron Ley et al. (2016), pero ésta no fue del todo homogénea, ya que cada uno de los peligros tuvo un comportamiento particular, tanto en el nivel de percepción alcanzado, como en el ritmo de pérdida o ganancia de visibilidad, como se describirá en las siguientes subsecciones.

\section{Nivel de visibilidad de los peligros}

En 2011, los peligros más visibles, es decir, aquellos identificados por más de la mitad de los encuestados, fueron siete: sismo, calor extremo, contaminación del aire, viento fuerte, frío extremo, plaga y agrietamiento del suelo (Figura $\mathrm{N}^{\circ} 1$ ). En 2016, tales peligros ocuparon también los primeros lugares de percepción, pero con una intensidad menor, y solo cuatro de ellos (sismo, calor, contaminación del aire y plaga) presentaron un nivel alto de visibilidad social.

Al inicio del periodo los peligros con los niveles más bajos de percepción (identificados por menos de $5 \%$ de los encuestados) fueron seis: maremoto, accidente aéreo, licuefacción, terrorismo, accidente ferroviario y radiación nuclear (Figura $\mathrm{N}^{\circ}$ 1); mientras que al final del periodo el 
número de amenazas de baja percepción aumentó a quince, adicionándose a las anteriores: flujo de lodo, deslizamiento, erupción volcánica, ciclón, lluvia intensa, granizada, sequía, explosión y fuga o derrame de sustancia química.

Cabe señalar que la mayoría de los peligros de alta visibilidad se caracterizan por su presencia constante en toda la ciudad, razón por la cual no resulta extraño que sean identificados por gran parte de los habitantes; mientras que la mayoría de los peligros de baja visibilidad tienen una probabilidad muy baja o nula de presentarse en el lugar y algunos de ellos (accidente ferroviario, licuefacción, deslizamiento, explosión y fuga de sustancia) amenazan sólo una parte de la superficie urbana o localizaciones específicas dentro de ella (Ley, 2019).

Esta situación hace evidente el papel que juega la experiencia cotidiana en la visibilidad de las amenazas, como producto de la oferta de estímulos del entorno, confirmando lo que señalan Durand (2008) y Qasim et al. (2015) sobre el papel del territorio en el mecanismo cognitivo que Tversky \& Kahneman (1974) denominan disponibilidad heurística.

Figura $\mathrm{N}^{\circ} 1$.

Porcentaje de personas que identificaron el peligro en el lugar en 2011 y 2016

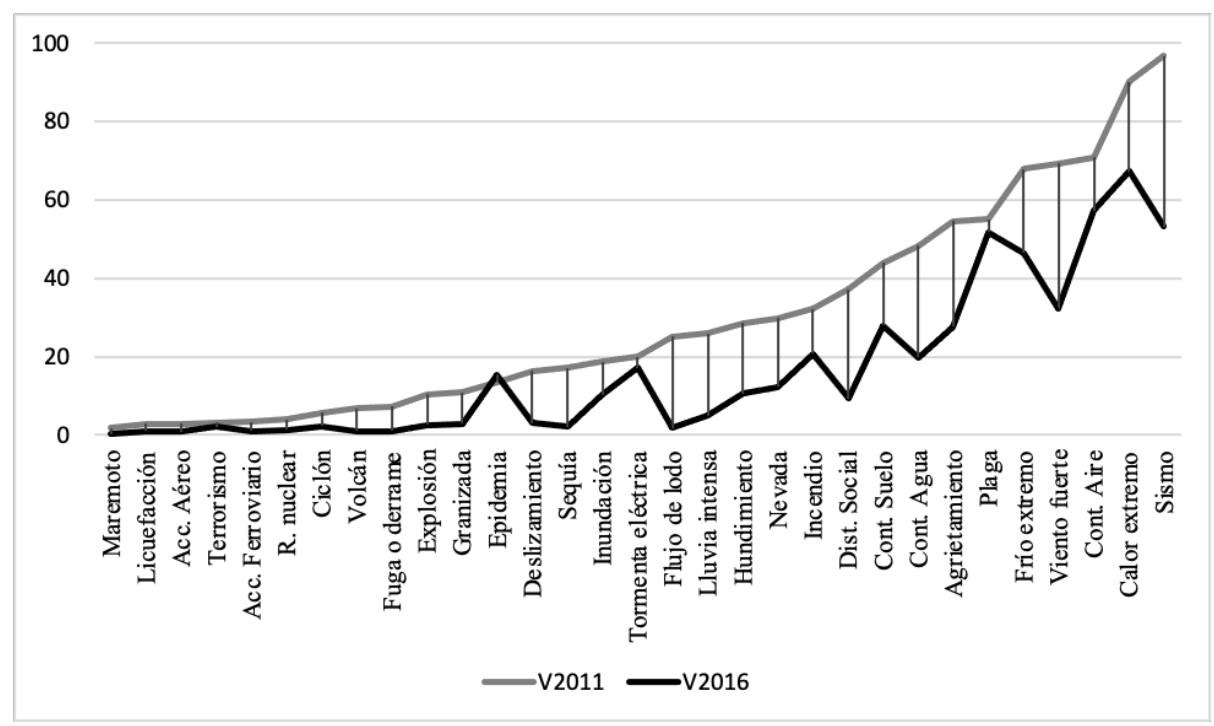

Fuente: Elaboración propia.

\section{Pérdida y ganancia de visibilidad}

La distancia entre las líneas de visibilidad de los peligros V2016 y V2011 (Figura N¹) revela la diferencia absoluta de la percepción en ambos años. Ésta es mayor en los sismos, vientos fuertes, contaminación del agua, disturbios sociales, agrietamiento del suelo, calor extremo, flujo de lodo, Iluvia intensa y frío extremo que, en las demás amenazas, con pérdidas que van del $20 \%$ al $44 \%$. El único peligro que incrementó su visibilidad fue la epidemia (2\%).

Algunos peligros fueron más dinámicos que otros en cuanto a la pérdida de visibilidad y de importancia. Para observar tal comportamiento, en la Figura $\mathrm{N}^{\circ} 2$ se muestran los peligros con 
respecto al número de posiciones ganadas o perdidas (eje de las abscisas) y al ritmo de cambio en la visibilidad (eje de ordenadas).

En general, como la visibilidad decreció, el ritmo de cambio presentó valores inferiores a uno (excepto en la epidemia). De tal forma que los peligros con valores cercanos a la unidad son los que disminuyeron más lentamente su visibilidad, como sucedió con la plaga, la contaminación del aire y la tormenta eléctrica $(0.8 \leq \mathrm{Cp}<1)$; mientras que los valores más próximos a cero, significan un decremento muy rápido en la percepción o visibilidad del peligro.

En esta última situación se encuentran el maremoto, la fuga o derrame de sustancia, la sequía, el flujo de lodo, la erupción volcánica, la lluvia intensa y el deslizamiento $(\mathrm{Cp}<0.2)$. El resto de los peligros experimentó un ritmo de cambio intermedio $(0.2 \leq \mathrm{Cp}<0.8)$, donde pueden distinguirse cambios rápidos $(0.2 \leq \mathrm{Cp}<0.4)$, moderados $(0.4 \leq \mathrm{Cp}<0.6)$ y lentos $(0.6 \leq \mathrm{Cp}<0.8)$.

El cambio de posición de los peligros presentó valores en un rango de -8 a 9 que indican la ganancia y pérdida de importancia con respecto a los demás peligros. De esta manera, en el eje de las abscisas se observa que la tormenta eléctrica, el terrorismo y el ciclón incrementaron su importancia relativa al ascender varias posiciones $(P \leq-4)$; mientras que la sequía, el disturbio social y el flujo de lodo, perdieron importancia al descender varias posiciones en el periodo $(P \geq 4)$.

En el caso opuesto se encuentran los peligros con movilidad muy baja o nula, que se desplazaron sólo una posición (deslizamiento, agrietamiento, hundimiento, nevada, calor extremo, radiación nuclear, contaminación del aire y accidente ferroviario) o ninguna (licuefacción, maremoto, frío extremo y accidente aéreo). En otras palabras, la importancia relativa de esos peligros no se alteró, independientemente del nivel de visibilidad y de cambio que alcanzaron en el periodo.

Figura $\mathrm{N}^{\circ} 2$.

Posición y ritmo de cambio de peligros en 2011 y 2016

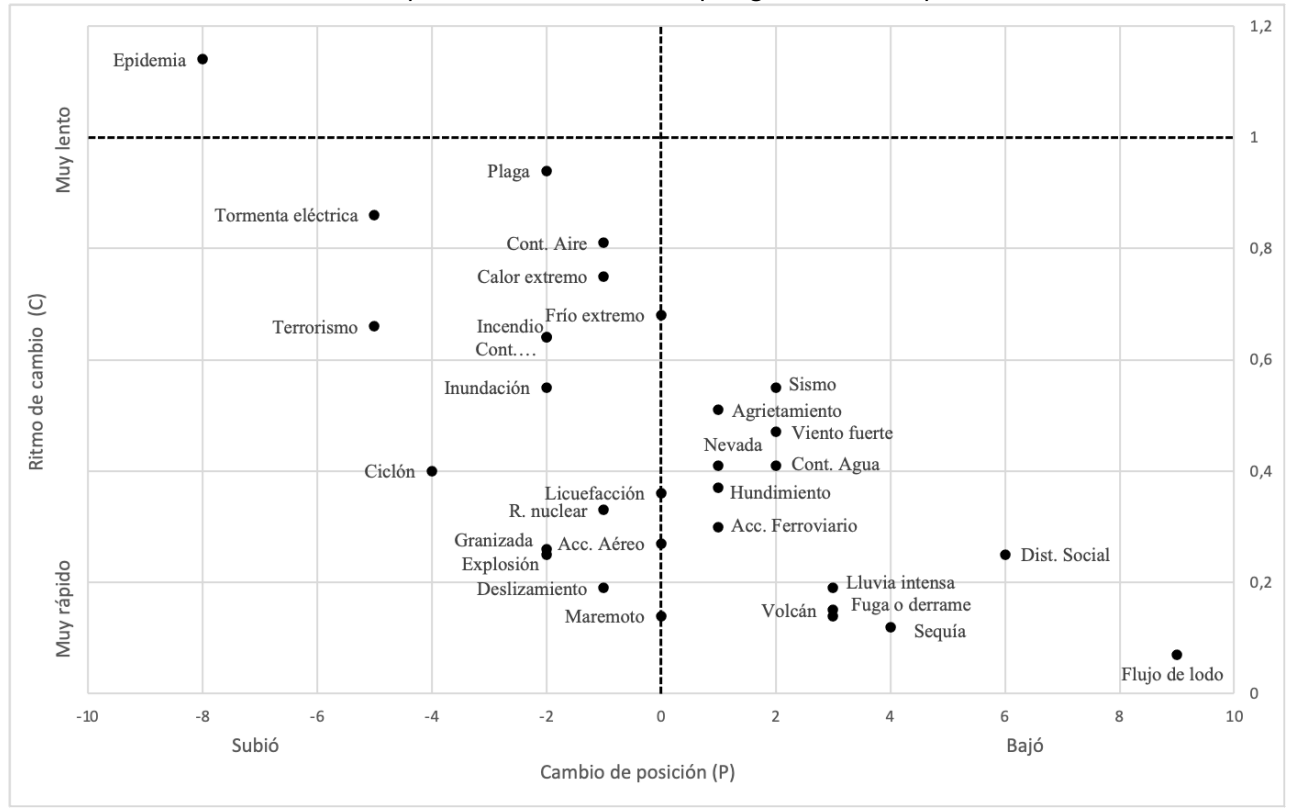

Fuente: Elaboración propia. 


\section{Peligros cambiantes y permanentes: los casos extremos}

En la combinación de ambas variables ( $\mathrm{Cp}$ y $\mathrm{P}$ ) y tomando en cuenta el nivel de visibilidad absoluta (cuadro 3), se identifica que el flujo de lodo, la sequía y el disturbio social fueron los peligros más dinámicos en cuanto al decremento en la percepción, por ende se trata de peligros cambiantes en descenso; mientras que la epidemia fue el único peligro que incrementó su visibilidad y aumentó considerablemente en importancia $(C p=1.14$ y $P=8)$, por tanto se considera un peligro cambiante en ascenso o de tipo emergente en la percepción social.

Esta dinámica perceptual ha sido reportada para peligros sanitarios emergentes como la influenza AH1N1 por Ibuka et al. (2010) y Fielding et al. (2014). Los primeros reportaron un aumento en la percepción y preocupación por el riesgo durante el surgimiento de la epidemia; los segundos observaron decaer la percepción, preocupación y medidas preventivas después de cinco años. Este proceso de amplificación inicial y atenuación posterior fue observado por Obenauer et al., (2018) para el caso del Ébola en un lapso de un año. Para peligros químico-tecnológicos (accidentes nucleares) Huang et al. (2013) y Susuki et al. (2018) reportaron la estabilización de la percepción con el paso del tiempo.

Por lo anterior, se entiende que la emergencia (sanitaria o nuclear) revela súbitamente la presencia del peligro en el lugar y su potencial catastrófico, dejando una marca particular (representatividad heurística); pero la preocupación disminuye conforme los estímulos dejan de alimentar la percepción.

Por su parte, la plaga y la contaminación del aire fueron los peligros menos dinámicos, ya que su visibilidad disminuyó muy lentamente y descendieron pocos lugares $(\mathrm{Cp} \geq 0.8$ y $\mathrm{P}<4)$, considerándose peligros constantes o permanentes en la percepción. En este perfil se pueden incluir las temperaturas extremas (calor y frío), el incendio y la contaminación del suelo, por su reducción lenta de visibilidad y desplazamiento de pocas posiciones $(0.6 \leq \mathrm{Cp}<0.8$ y $\mathrm{P}<4)$. Este grupo es permanentemente percibido en el lugar, pues presenta niveles alto y muy alto de percepción.

En este sentido, los peligros cambiantes en Mexicali presentan dos momentos perceptuales: la amplificación inicial (epidemia) y la atenuación posterior (flujo de lodo, sequía y disturbio social); que puede explicarse por la oferta súbita de información en peligros emergentes y la pérdida de ésta en los que son atenuados. Mientras que los peligros constantes, como se señaló previamente, se mantienen permanentemente visibles por su experiencia cotidiana en el lugar.

Cuadro $\mathrm{N}^{\circ} 3$.

Resumen de los peligros más cambiantes y constantes

\begin{tabular}{|l|l|l|l|l|l|l|l|l|}
\hline \multirow{2}{*}{ Peligro } & \multirow{2}{*}{ Tipo } & \multirow{2}{*}{ Subtipo } & \multicolumn{2}{|l|}{ Visibilidad } & \multirow{2}{*}{ Cp } & \multicolumn{2}{|l|}{ Posición } & \multirow{2}{*}{ P } \\
& & & V2011 & V2016 & & P2011 & P2016 & \\
\hline Epidemia & Cambiante & Ascendente & 13.51 & 15.38 & 1.14 & 20 & 12 & -8 \\
\hline Disturbio social & Cambiante & Descendente & 37.14 & 9.23 & 0.25 & 10 & 16 & 6 \\
\hline Flujo de lodo & Cambiante & Descendente & 25.19 & 1.79 & 0.07 & 15 & 24 & 9 \\
\hline Sequía & Cambiante & Descendente & 17.14 & 2.05 & 0.12 & 18 & 22 & 4 \\
\hline Plaga & Constante & Visible & 55.06 & 51.79 & 0.94 & 6 & 4 & -2 \\
\hline Contaminación del aire & Constante & Visible & 70.91 & 57.18 & 0.81 & 3 & 2 & -1 \\
\hline
\end{tabular}




\begin{tabular}{|l|l|l|l|l|l|l|l|l|l|}
\hline \multirow{2}{*}{ Peligro } & \multirow{2}{*}{ Tipo } & \multirow{2}{*}{ Subtipo } & \multicolumn{2}{|l|}{ Visibilidad } & \multirow{2}{*}{ Cp } & \multicolumn{3}{|l|}{ Posición } & \multirow{2}{*}{ P } \\
& & & V2011 & V2016 & & P2011 & P2016 & \\
\hline Calor & Constante2 & Visible & 90.13 & 67.18 & 0.75 & 2 & 1 & -1 \\
\hline Frío & Constante2 & Visible & 67.79 & 46.41 & 0.68 & 5 & 5 & 0 \\
\hline Incendio & Constante2 & Visible & 32.21 & 20.77 & 0.64 & 11 & 9 & -2 \\
\hline Contaminación del suelo & Constante2 & Visible & 43.90 & 27.95 & 0.64 & 9 & 7 & -2 \\
\hline
\end{tabular}

Fuente: Elaboración propia.

El Cuadro N4 muestra el comportamiento de los anteriores peligros en el periodo a partir de indicadores de la manifestación física de las amenazas en los momentos previos a cada encuesta y del cociente de cambio (Co).

\section{- Cambiantes}

En los peligros cambiantes, las personas que participaron en la encuesta e identificaron las epidemias, hicieron referencia principalmente al brote de rickettsiosis (transmitida por garrapatas) (UABC, 2016), cuyo primer caso en Baja California se presentó en Mexicali durante el año 2009. Desde entonces, la tasa estatal de mortalidad se ha incrementado hasta duplicarse en 2016 (cuadro 4), con el mayor número de casos y defunciones ocurridos en Mexicali (COPLADE, 2017; ISESALUD, 2016). Así, el aumento en la percepción de este peligro puede tener una base objetiva, aunque es menor $(\mathrm{Cp}=1.88)$ que el ocurrido en la manifestación objetiva del peligro $(\mathrm{Co}=2.19)$.

Por su parte, los principales disturbios sociales que los encuestados reportaron son pleitos entre vecinos, delincuencia y pandillerismo (UABC, 2016), y no aquellos de interés en materia de protección civil como son las marchas, mítines, bloqueos, amotinamientos, entre otros eventos relacionados con la alta concentración de población, sabotaje y actos masivos violentos. En términos generales, la incidencia delictiva se mantuvo constante en el periodo, con un leve decremento en los años previos a la encuesta de 2016 (Co = 0.90); esto es congruente con la disminución en la percepción, pero no con el ritmo acelerado de ésta $(C p=0.25)$.

Con respecto a la sequía, según el índice normalizado de precipitación pluvial disponible para Mexicali (CONAGUA, 2018), en casi todos los meses del año 2007, así como en diciembre de 2009 y enero de 2010 se presentaron episodios extremadamente y excepcionalmente secos (sequía alta y muy alta); posteriormente sólo se presentaron episodios moderadamente secos en 2015 y 2016. En otras palabras, la intensidad de la sequía disminuyó considerablemente en el segundo período, tal como lo percibe la población.

En cambio, el flujo de lodo o alud no tiene presencia objetiva en la ciudad, se trata de un peligro que fue amplificado o magnificado en la percepción al inicio del período y que se desvaneció posteriormente. Esta situación puede relacionarse con los procesos de licuefacción, estancamiento de agua e inundaciones parciales que fueron detonados por el sismo fuerte de 2010 (UABC, AM \& SEDESOL, 2011).

\section{- Permanentes}

Con respecto a los peligros constantemente visibles, si bien, las plagas son un problema exclusivo del sector agrícola, la población urbana las entiende como sinónimo de fauna nociva, por ello identifica como amenazas a trece especies, de las que sobresalen en orden de importancia: 
el mosquito, la garrapata, la cucaracha y la mosca (UABC, 2016), cuya presencia es permanente en la ciudad.

Por su parte, como Mexicali es una de las ciudades mexicanas con los niveles más altos de contaminación del aire (INECC, 2017) se entiende que este peligro sea constantemente visible o perceptible para los habitantes. Cabe señalar que los datos locales disponibles no ofrecen el panorama completo de la evolución de la contaminación en el periodo (GEBC \& SEMARNAT, 2018). Pero, si se toman como referencia los valores de contaminación de la vecina ciudad de Calexico, se observa que, de los contaminantes criterio, solo las partículas suspendidas $\mathrm{PM}_{10}$ y $\mathrm{PM}_{2.5}$ excedieron los parámetros normativos en varios años. Las $\mathrm{PM}_{10}$ sobresalieron en la primera parte del periodo y las $\mathrm{PM}_{2.5}$ en la segunda, por tanto, se considera que no hubo cambios importantes en la manifestación física del fenómeno como tampoco sucedió con la percepción.

Cuadro $\mathrm{N}^{\circ} 4$.

Manifestación de los peligros en el lugar durante el periodo 2007 a 2016

\begin{tabular}{|c|c|c|c|c|c|}
\hline Peligro & Indicadores & 2007-2011 & 2012-2016 & Co & Fuente \\
\hline Epidemia & $\begin{array}{l}\text { Tasa estatal de mortalidad } \\
\text { por rickettsiosis en BC } \\
\left({ }^{*} 2009 y^{* *} 2016\right) \text {. }\end{array}$ & $0.27^{*}$ & 0.59 ** & 2.19 & $\begin{array}{l}\text { COPLADE (2017); } \\
\text { ISESALUD (2016). }\end{array}$ \\
\hline Delitos & $\begin{array}{l}\text { Porcentaje con respecto al } \\
\text { total del periodo. }\end{array}$ & $51 \%$ & $49 \%$ & 0.90 & GESI(2019) \\
\hline \multirow[t]{2}{*}{ Sequía } & $\begin{array}{l}\text { Porcentaje de meses con } \\
\text { algún nivel de sequía. }\end{array}$ & $21.7 \%$ & $6.7 \%$ & 0.31 & $\begin{array}{l}\text { CONAGUA (2018). } \\
\text { Estación } 2033\end{array}$ \\
\hline & $\begin{array}{l}\text { Número de meses extre- } \\
\text { mada y excepcionalmente } \\
\text { secos. }\end{array}$ & 12 & 0 & - & $\begin{array}{l}\text { CONAGUA (2018). } \\
\text { Estación } 2033\end{array}$ \\
\hline F. de lodo & No está presente. & N.A. & N.A. & N.A. & N.A. \\
\hline Plaga & $\begin{array}{l}\text { Amenazan al sector agrí- } \\
\text { cola. }\end{array}$ & N.A. & N.A. & N.A. & N.A. \\
\hline \multirow[t]{2}{*}{$\begin{array}{l}\text { Contaminación } \\
\text { del aire }\end{array}$} & \begin{tabular}{|l|} 
Porcentaje de días que \\
exceden un promedio diario \\
de $65 \mu \mathrm{g} / \mathrm{m}^{3}$ de $\mathrm{PM}_{25}$
\end{tabular} & $0.6 \%$ & $2.2 \%$ & 3.9 & \multirow[t]{2}{*}{$\begin{array}{l}\text { CARB (2018). } \\
\text { Estación 13698 } \\
\text { Ethel, Calexico }\end{array}$} \\
\hline & \begin{tabular}{|l|} 
Porcentaje de días que \\
exceden un promedio diario \\
de $120 \mu \mathrm{g} / \mathrm{m}^{3}$ de $\mathrm{PM}_{10}$. \\
\end{tabular} & $3.0 \%$ & $2.5 \%$ & 0.82 & \\
\hline Calor & $\begin{array}{l}\text { Rango de temperatura } \\
\text { máxima }\left({ }^{\circ} \mathrm{C}\right) .\end{array}$ & 46.7 a 47.8 & 46.7 a 48.9 & 1.02 & $\begin{array}{l}\text { NOAA (2018). Es- } \\
\text { tación El Centro } \\
23199\end{array}$ \\
\hline Frío & $\begin{array}{l}\text { Rango de temperatura míni- } \\
\text { ma }\left({ }^{\circ} \mathrm{C}\right) \text {. }\end{array}$ & -0.6 a -7.2 & -0.6 a -3.9 & 0.54 & $\begin{array}{l}\text { NOAA (2018). Es- } \\
\text { tación El Centro } \\
23199\end{array}$ \\
\hline Incendio & $\begin{array}{l}\text { Porcentaje con respecto al } \\
\text { total del período. }\end{array}$ & $45 \%$ & $55 \%$ & 1.22 & $\begin{array}{l}\text { C O P L A D E M M } \\
(2011 ; \quad 2017) \text {, } \\
\text { UABC, AM y SE- } \\
\text { DESOL (2011) }\end{array}$ \\
\hline $\begin{array}{l}\text { Contaminación } \\
\text { del suelo }\end{array}$ & No hay datos. & S.D. & S.D. & S.D. & S.D. \\
\hline
\end{tabular}

Fuente: Elaboración propia.

Al ampliar el listado de los peligros permanentes para incluir aquellos que experimentaron un ritmo de cambio intermedio de tipo lento $(0.6 \leq \mathrm{Cp}<0.8)$, se incorporaron las temperaturas extre- 
mas, los incendios y la contaminación del suelo (constante2 en el cuadro 3), cuya manifestación en la ciudad se describe en los párrafos subsecuentes.

A nivel global las temperaturas extremas cambiaron durante el periodo con una intensificación del calor y una disminución del frío (OMM, 2016). Según datos del NOAA ${ }^{3}$ (2018), en los años previos a la segunda encuesta hubo un incremento en los valores de la temperaturas máxima y mínima, es decir, aumentó el calor y disminuyó el frío en la zona. La misma fuente revela que el año 2016 fue el más cálido y el 2011 el más frío. De esta manera, es comprensible que la visibilidad absoluta del frío disminuyera y que lo hiciera a un ritmo lento, pero no así la del calor que, a pesar de intensificarse en el lugar, perdió lentamente visibilidad.

Por su parte, los incendios perdieron visibilidad y presentaron un cambio relativamente lento $(\mathrm{Cp}=0.64)$, aunque el número de éstos fue mayor durante la segunda parte del periodo $(\mathrm{Co}=1.22)$. Es decir, a pesar de que aumentó el número de incendios en la ciudad, este peligro se mantuvo constante en la percepción, quizá debido al comportamiento espacial y temporalmente selectivo de estos eventos químicos.

Por último, la contaminación del suelo disminuyó lentamente su percepción en el periodo. Esto a pesar de que más de una tercera parte de los encuestados consideran que el problema de la basura en la ciudad ha aumentado, además de relacionar este asunto con el incremento en la cantidad de lotes baldíos y casas abandonadas, e identificar la proliferación de fauna nociva como una de las consecuencias principales (UABC, 2016).

\section{El resto de los peligros}

El resto de los peligros presentó un ritmo de cambio intermedio de moderado $(0.4 \leq \mathrm{Cp}<0.6)$ a rápido $(0.2 \leq \mathrm{Cp}<0.4)$, desplazándose 3 o menos posiciones, con excepción de la tormenta eléctrica, el terrorismo y el ciclón que se desplazaron 4 o más posiciones. Aunque no ocuparon las posiciones extremas de permanencia y cambio, se aproximaron a algunas de ellas, presentando características diversas.

El grupo de peligros de descenso intermedio-rápido en la percepción incluye: ciclón, radiación nuclear, accidente aéreo, granizada, licuefacción, explosión, accidente ferroviario y hundimiento. Se caracteriza por una percepción muy baja (V2016 < 5\%), excepto el hundimiento que presentó un nivel bajo (10.51\%). Esto corresponde con su presencia en la ciudad, ya que los tres primeros tienen una probabilidad nula o muy baja de ocurrir, mientras que el resto está presente en zonas específicas o reducidas del espacio urbano. Así, se entiende que la rápida pérdida de visibilidad se relaciona con la amplificación de la que fueron objeto al inicio del período y no con su manifestación física en el lugar.

Se utilizó la información de la Estación El Centro, California debido a que los datos para las estaciones del municipio de Mexicali del Sistema de Información para el Manejo del Agua de Riego en Baja California están incompletos y presentan errores en varios años. 
Por su parte, el grupo de las amenazas de descenso intermedio-moderado incluye: sismo, viento fuerte, agrietamiento de suelo, inundación, contaminación del agua y nevada. Estos peligros son relativamente constantes, pero muy distintos entre sí, ya que los tres primeros son de visibilidad social alta o muy alta, y el resto de visibilidad baja. Pero, a diferencia del sismo y el viento fuerte, cuya manifestación es recurrente en toda la ciudad, el resto se restringe a ciertas zonas o resulta improbable de ocurrir.

La dinámica moderada de cambio de los peligros de esta subsección puede expresar una tendencia a permanecer constantemente visibles o invisibles; pero, en el período, peligros relevantes como el sismo y el viento fuerte dejaron de ser identificados por más de una tercera parte de la población, lo que puede derivar en el relajamiento de las prácticas de seguridad en los hogares, como se observó en otros estudios.

\section{Consideraciones finales}

Acerca de la permanencia y cambio en la percepción de los peligros por parte de los habitantes de la ciudad de Mexicali se observó que en el periodo disminuyó de manera importante el número de peligros identificados en el lugar, como también sucedió con la visibilidad de casi todos ellos, mostrando una fase de atenuación generalizada.

En el quinquenio previo los peligros en estado latente se hicieron evidentes, algunos de ellos provocando daños importantes, lo que conduce a pensar que, con la posterior disminución en la oferta de estímulos, los peligros empezaron a ocultarse y, con ello, se moderó la amplificación que sufrieron algunos en 2011. Este proceso también afectó la visibilidad de las amenazas relevantes del lugar, cuya atenuación paulatina puede resultar en la reducción de medidas preventivas y prácticas de seguridad en los hogares.

En este sentido, la temporalidad de la percepción tiene que ver con la forma en que cada evento se presenta en el lugar, donde los peligros más frecuentes y que amenazan toda la ciudad son reconocidos por más personas (visibles) que aquellos de baja frecuencia y localización selectiva (invisibles).

No obstante, como ambos tipos de peligros se mantuvieron relativamente constantes en la percepción social se entiende que la frecuencia del evento por sí sola no explica los cambios perceptuales, sino que éstos se enmarcan en la posibilidad de que adquieran rápidamente visibilidad cuando se rompe de manera abrupta el statu quo o de que se vuelvan invisibles nuevamente porque retornan a ello.

Tal situación remite al papel del entorno como unidad perceptiva que enmarca la experiencia cotidiana del habitante y, con ello, a la importancia que tiene la experiencia directa en el aprendizaje social del riesgo, aspectos sobre los que existe un amplio campo para la investigación. Sin dejar de lado el papel que suelen jugar los medios de comunicación en la construcción de la percepción social y en los sesgos que la caracterizan. 


\section{Referencias}

CARB. Air Quality and Emissions. California Air Resources Board, 2018. (Consulta: 20/08/2018). https://www.arb.ca.gov/html/ds.htm

CARDOSO, M.M.(2020). Estudio de la vulnerabilidad y la resiliencia en la ciudad de Santa Fe, Argentina: El rol de los servicios urbanos en general y del transporte de pasajeros en particular. Revista de Geografía Norte Grande, No 73, p. 133-159. https://doi.org/10.4067/S0718-34022019000200133

CENAPRED. Sistema de información geográfica sobre riesgos. Centro Nacional de Prevención de Desastres, 2018. (Consulta: 25/02/2018). http://www.atlasnacionalderiesgos.gob.mx/archivo/visor-capas.html

CFE. Manual de diseño de obras civiles. Distrito Federal: Comisión Federal de Electricidad, 2008.

CONAGUA. Índice estandarizado de precipitación pluvial. Comisión Nacional del Agua, 2018. (Consulta: 12/08/2018). https://smn.conagua.gob.mx/es/climatologia/monitor-de-sequia/spi

COMPLADEMM. Anuario estadístico 2011. Mexicali: Comité de Planeación para el Desarrollo Municipal de Mexicali, 2011.

COMPLADEMM. Anuario estadístico municipal 2017. Mexicali: Comité de Planeación para el Desarrollo Municipal de Mexicali, 2017.

COPLADE. Actualización. Programa Sectorial de Salud 2015-2019. Mexicali: Comité de Planeación para el Desarrollo del Estado, 2017.

COVELLO, V.T. The perception of technological risks: a literature review. Technological Forecasting and Social change, 1983, Vol. 23, No 4, p. 285-297. https://doi.org/10.1016/0040-1625(83)90032-X

CUTTER, S.L. Living with risk. London; New York: Edward Arnold; Routledge, 1993.

DURAND, L. De las percepciones a las perspectivas ambientales. Una reflexión teórica sobre la antropología y la temática ambiental. Nueva antropología, 2008, Vol. 2, № 68, p. 75-87. Disponible en http://www.scielo.org.mx/scielo.php?script=sci_arttext\&pid=S0185-06362008000100005

ESTRADA, G. Prevenir riesgos o atender desastres en las ciudades. Dos opciones de política con alcances distintos. Trace, 2009, No 56, p. 41-56. Disponible en https://journals.openedition.org/ trace/1431

EIRD. 2009 UNISDR. Terminología sobre reducción del riesgo de desastres. Ginebra: Estrategia Internacional para la Reducción de Desastres, 2009.

FIELDING, R., COWLING, B.J., LIAO, Q., \& LAM, W.W. Behavioural changes in relation to risk perception and prevention of avian and human influenza in Hong Kong, 2006 to 2010. Hong Kong 
Medical Journal, 2014, Vol. 20, N4, p. 26-28. Disponible en https://www.hkmj.org/system/files/ hkm1404sp4p26_0.pdf

FINUCANE, M.L., ALHAKAMI, A., SLOVIC, P., \& JOHNSON, S.M. The affect heuristic in judgments of risk and benefits. Journal of Behavioral Decision Making, 2000, Vol. 13, No 1, p. 1-17. https://doi. org/10.1002/(SICI)1099-0771(200001/03)13:1<1::AID-BDM333>3.0.CO;2-S

FOLKES, V. S. The availability heuristic and perceived risk. Journal of Consumer Research, 1988, Vol. 15, No1, p.13-23. https://doi.org/10.1086/209141

GEBC \& SEMARNAT. Programa para Mejorar la Calidad del Aire del Estado de Baja California, Mexicali 2018-2027. Mexicali: Gobierno del Estado de Baja California; Secretaría de Medio Ambiente y Recursos Naturales, 2018.

GREGORY, R.S. \& SATTERFIELD, T.A. Beyond perception: The experience of risk and stigma in community contexts. Risk Analysis, 2002, Vol. 22, No 2, p. 347-358. https://doi.org/10.1111/02724332.00017

HUANG, L., ZHOU, Y., HAN, Y., HAMMITT, J.K., BI, J., \& LIU, Y. Effect of the Fukushima nuclear accident on the risk perception of residents near a nuclear power plant in China. Proceedings of the National Academy of Science of the United States of America, 2013, Vol. 110, № 49, p.19742-19747. https://doi.org/10.1073/pnas.1313825110

IBUKA, Y., CHAMPAN, G.B., MEYERS, L.A., LI, M., \& GALVANI, A.P. The dynamics of risk perceptions and precautionary behavior in response to 2009 (AH1N1) pandemic influenza. BMC Infectious Diseases, 2010, Vol. 10, № 296, p. 1-11. https://doi.org/10.1186/1471-2334-10-296

INECC. Informe Nacional de Calidad del Aire 2016, México. Ciudad de México: Instituto Nacional de Ecología y Cambio Climático, 2017.

INEGI. Censo de Población y Vivienda 2010. Aguascalientes: Instituto Nacional de Estadística y Geografía, 2010.

ISESALUD. Panorama epidemiológico. Rickettsiosis en Baja California año 2016. Mexicali: Dirección General de Información en Salud, Subsistema Especial de Vigilancia Epidemiológica. Instituto de Servicios de Salud Pública de Baja California, 2016.

KASPERSON, R.E. \& KASPERSON, J.X. The social amplification and attenuation of risk. The ANNALS of the American Academy of Political and Social Science, 1996, Vol. 545, № 1, p. 95-105. https://doi. org/10.1177/0002716296545001010

KASPERSON, R.E., RENN, O. SLOVIC, P., BROWN, H.S., EMEL, J., GOBLE, R., KASPERSON, J.X., \& RATICK, S. The social amplification of risk: A conceptual framework. Risk Analysis, 1988, Vol. 8, No 2, p. 177-187. https://doi.org/10.1111/j.1539-6924.1988.tb01168.x 
LEY, J. La producción del espacio como riesgo. Mexicali: UABC, 2011.

LEY, J. Evaluación multi peligro de las instalaciones críticas en la ciudad de Mexicali. En: LEY, J. y MAS, J.F. (coords.). Análisis geoespacial en los estudios urbanos. Mexicali: UABC, UNAM, Selper, 2019, p. 201-217.

LEY, J., DENEGRI, F.M., ORTEGA, L.M. Spatial dimension of urban hazardscape perception: The case of Mexicali, Mexico. International Journal of Disaster Risk Reduction, 2015, Vol. 14, No 4, p. 487-495. https://doi.org/10.1016/j.ijdrr.2015.09.012

LEY, J., DENEGRI, F.M. \& SÁNCHEZ, G. Peligros constantes y cambiantes en la percepción social del paisaje de amenazas en la ciudad de Mexicali. Región y Sociedad, 2016, Vol. 28, N 66, p. 269-294. https://doi.org/10.22198/rys.2016.66.a199

LEY, J., ORTEGA, L.M., \& DENEGRI, F.M. Diferencias en la percepción de peligros múltiples entre los habitantes de una ciudad fronteriza de México. Estudios Demográficos y Urbanos, 2019, Vol. 34, № 1, p. 71-99. https://doi.org/10.24201/edu.v34i1.1737

MACLEOD, C. \& CAMPBELL, L. Memory accessibility and probability judgments: an experimental evaluation of the availability heuristic. Journal of Personality and Social Psychology, 1992, Vol. 63, $\mathrm{N}^{\circ}$ 6, p. 890-902. https://doi.org/10.1037//0022-3514.63.6.890

MAÑEZ, M., CARMONA, M., HARO, D., \& HANGER, S. Risk perception. En: AERTS, J. y MYSIAK, J. (eds.). Novel Multi-Sector Partnerships in Disaster Risk Management. Results of the ENHANCE project. Bruselas: EU FP7 project ENHANCE, 2016, p. 51-67.

MARGOLIS, H. Dealing with risk: Why the public and the experts disagree on environmental issues. Chicago: University of Chicago Press, 1996.

México. Ley General de Protección Civil. Diario Oficial de la Federación, 6 de junio de 2012, p. 29.

NOAA. National Centers for Environmental Information. National Oceanic and Atmospheric Administration, 2018. (Consulta: 13/09/2018). https://www.ncdc.noaa.gov/

OBENAUER, J., RÜBSAMEN, N., GARSEVANIDZE, E., KARCH, A., \& MIKOLAJCZYK, R.T. Changes in risk perceptions during the 2014 Ebola virus disease epidemic: results of two consecutive surveys among the general population in Lower Saxony, Germany. BMC Public Health, 2018, Vol. 18, $\mathrm{N}^{\circ}$ 628, p. 1-8. https://doi.org/10.1186/s12889-018-5543-1

ONU. Decenio internacional para la reducción de los desastres naturales. (A/RES/42/169). Asamblea General, Organización de las Naciones Unidas, 1987.

ONU. Informe de la Conferencia Mundial sobre la Reducción de los Desastres Naturales (A/CONF. 172/9). Yokohama: Organización de las Naciones Unidas, 1994. 
ONU. Informe de la Conferencia Mundial sobre la Reducción de los Desastres. (A/CONF.206/6). Kobe, Hyogo: Organización de las Naciones Unidas, 2005.

ONU. Marco de Sendai para la Reducción del Riesgo de Desastre 2015-2030. Conferencia Mundial Sobre la Reducción de Desastres. Ginebra: Organización de las Naciones Unidas, 2015a.

ONU. Aplicación de la Estrategia Internacional para la Reducción de los Desastres. Informe del Secretario General. (A/70/282). Asamblea General, Organización de las Naciones Unidas 2015b.

ONU. Nueva Agenda Urbana. Quito: Organización de las Naciones Unidas, 2017.

OMM. Estado del clima mundial en 2011-2015. Ginebra: Organización Meteorológica Mundial, 2016.

PACHUR, T., HETWIG, R., \& STEINMANN, F. How do people judge risks: Availability heuristic, affect heuristic, or both? Journal of Experimental Psychology: Applied, 2012, Vol. 18, No 3, p. 314-330. https://doi.org/10.1037/a0028279

PIDGEON, N., KASPERSON, R.E., \& SLOVIC, P. The social amplification of risk. Cambridge: Cambridge University Press, 2003.

QASIM, S., KHAN, A., SHRESTHA, R., \& QASIM, M. Risk perception of the people in the flood prone Khyber Pukhthunkhwa province of Pakistan. International Journal of Disaster Risk Reduction, 2015, Vol. 14, N 4, p. 373-378. https://doi.org/10.1016/j.ijdrr.2015.09.001

RENN, O. Risk perception and communications: Lessons for the food and food packaging industry. Food Additives and Contaminants, 2005, Vol. 22, No 10, p. 1061-1071. https://doi. org/10.1080/02652030500227792

ROMANUS, J., HASSING, L., \& GARLING, T. A loss-sensitivity explanation of integration of prior outcomes in risky decisions. Acta Psychologica, 1996, Vol. 93, № 1-3, p. 173-183. https://doi. org/10.1016/0001-6918(96)00007-8

SADIQ, A.A. THARP, K., GRAHAM, J.D., \& TYLER, J. Temporal stability and changes in risk perception rankings of hazardous activities and technologies. Journal of Risk Research, 2017, Vol. 22, No 1, p. 93-109. https://doi.org/10.1080/13669877.2017.1351474

SHEAFFER, R.L., MENDENHALL, W., \& OTT, R.L. Elementos de muestreo. Distrito Federal: Grupo Editorial Iberoamérica, 1987.

SHERMAN, S.J., CIALDINI, R.B., SCHWARTZMAN, D.F., \& REYNOLDS, K.D. Imagining Can Heighten or Lower the Perceived Likelihood of Contracting a Disease: The Mediating Effect of Ease of Imagery. Personality and Social Psychology Bulletin, 1985, Vol. 11, No 1, p. 118-127. https://doi. org/10.1177/0146167285111011

SLOVIC, P. The perception of risk. London: Earthscan, 2000. 
GESI. Estadísticas delictivas. Bases de datos de incidencia delictiva. Guardia Estatal de Seguridad e Investigación, 2019. (Consulta: 25/01/2019). https://www.seguridadbc.gob.mx/contenidos/estadisticas5.php

STOFFLE, R.W., TRAUGOTT, M.W., STONE, J.V., MCINTYRE, P.D., JENSEN, F.V., \& DAVIDSON, C.C. Risk perception mapping: Using ethnography to define the locally affected population for a low - level radioactive waste storage facility in Michigan. American Anthropologist, 1991, Vol. 93, № 3, p. 611635. https://doi.org/10.1525/aa.1991.93.3.02a00050

SUZUKI, Y., TAKEBAYASHI, Y., YASUMURA, S., MURAKAMI, M., HARIGANE, M., YABE, H., OHIRA, T., OHTSURU, A., NAKAJIMA, S., \& MAEDA, M. Changes in Risk Perception of the Health Effects of Radiation and Mental Health Status: The Fukushima Health Management Survey. International Journal of Environmental Research and Public Health, 2018, Vol. 15, № 6, p. 1-11. https://doi. org/10.3390/ijerph15061219

TVERSKY, A. \& KAHNEMAN, D. Availability: A heuristic for judging frequency and probability. Cognitive Psychology, 1973, Vol. 5, Nº 2, p. 207-232. https://doi.org/10.1016/0010-0285(73)90033-9

TVERSKY, A. \& KAHNEMAN, D. Judgment under uncertainty: Heuristics and biases. Science, 1974, Vol. 185, N 4157, p. 1124-1131. Disponible en http://www.jstor.org/stable/1738360.

TYLER, T. R. \& COOK, F. L. The mass media and judgments of risk: Distinguishing impact on personal and societal level judgments. Journal of Personality and Social Psychology, 1984, Vol. 47, $\mathrm{N}^{\circ} 4$, p. 693-708. https://doi.org/10.1037/0022-3514.47.4.693

UABC, AM, \& SEDESOL. Atlas de riesgos del municipio de Mexicali. Actualización 2011. Mexicali: Universidad Autónoma de Baja California, Ayuntamiento de Mexicali y Secretaría de Desarrollo Social, 2011.

UABC. Percepción local del riesgo. [Base de datos de la encuesta]. Mexicali: Instituto de Investigaciones Sociales, Universidad Autónoma de Baja California, 2011.

UABC. Percepción local de peligros: ciudad de Mexicali. [Base de datos de la encuesta]. Mexicali: Instituto de Investigaciones Sociales, Universidad Autónoma de Baja California, 2016.

WISNER, B., BLAIKIE, P., CANNON, T., \& DAVIS, I. At Risk. Natural hazards, people's vulnerability and disasters (2da. Ed.). London; New York: Routledge, 2004. 